\title{
Potential of LHC to determine the Dark Matter properties in the IDM
}

\author{
Dorota Sokołowska*i \\ University of Warsaw, Faculty of Physics, Hoza 69, 00-681 Warsaw, Poland \\ E-mail: dsokefuw.edu.pl
}

The Inert Doublet Model (IDM) can provide a viable Dark Matter candidate in agreement with current collider and relic density constraints in three regions of DM mass: $M_{D M} \lesssim 10 \mathrm{GeV}$, $40 \mathrm{GeV} \lesssim M_{D M} \lesssim 160 \mathrm{GeV}$ and $M_{D M} \gtrsim 500 \mathrm{GeV}$. Further constraints for the DM candidate can come from direct and indirect detection experiments. However, as for now there is no agreement how to consistently interpret various reported signals and the exclusion limits.

We discuss combined constraints on scalar DM from the IDM coming from the LHC Higgs data, namely the Higgs diphoton decay channel, and WMAP relic density measurements. They lead to an exclusion of a large part of the IDM parameter space, setting limits on DM that are stronger or comparable to these obtained by the DM detection experiments.

The European Physical Society Conference on High Energy Physics -EPS-HEP2013

18-24 July 2013

Stockholm, Sweden

\footnotetext{
${ }^{*}$ Speaker.

${ }^{\dagger}$ This work was supported in part by the grant NCN OPUS 2012/05/B/ST2/03306 (2012-2016).
} 


\section{Introduction}

The Inert Doublet Model (IDM) is one of the simplest extensions of the Standard Model (SM) that can provide a Dark Matter (DM) candidate in agreement with astrophysical data. The scalar sector in the IDM consists of a SM-like Higgs doublet $\Phi_{S}$ and a second $S U(2)$ doublet, $\Phi_{D}$, which is odd under a $D\left(Z_{2}\right)$ symmetry: $\Phi_{S} \rightarrow \Phi_{S}, \Phi_{D} \rightarrow-\Phi_{D}$, SM fields $\rightarrow$ SM fields [1].

The IDM is defined as a $2 \mathrm{HDM}$ with a $D$-symmetric potential and vacuum state:

$$
\begin{gathered}
V=-\frac{1}{2}\left[m_{11}^{2}\left(\Phi_{S}^{\dagger} \Phi_{S}\right)+m_{22}^{2}\left(\Phi_{D}^{\dagger} \Phi_{D}\right)\right]+\frac{\lambda_{1}}{2}\left(\Phi_{S}^{\dagger} \Phi_{S}\right)^{2}+\frac{\lambda_{2}}{2}\left(\Phi_{D}^{\dagger} \Phi_{D}\right)^{2} \\
+\lambda_{3}\left(\Phi_{S}^{\dagger} \Phi_{S}\right)\left(\Phi_{D}^{\dagger} \Phi_{D}\right)+\lambda_{4}\left(\Phi_{S}^{\dagger} \Phi_{D}\right)\left(\Phi_{D}^{\dagger} \Phi_{S}\right)+\frac{\lambda_{5}}{2}\left[\left(\Phi_{S}^{\dagger} \Phi_{D}\right)^{2}+\left(\Phi_{D}^{\dagger} \Phi_{S}\right)^{2}\right], \\
\left\langle\Phi_{S}\right\rangle=\frac{1}{\sqrt{2}}\left(\begin{array}{l}
0 \\
v
\end{array}\right), \quad\left\langle\Phi_{D}\right\rangle=\frac{1}{\sqrt{2}}\left(\begin{array}{l}
0 \\
0
\end{array}\right), \quad v=246 \mathrm{GeV},
\end{gathered}
$$

as well as the Yukawa interaction set to Model I, so that only $\Phi_{S}$ couples to fermions [1].

In the IDM only one doublet, $\Phi_{S}$, is involved in the EW symmetry breaking. $\Phi_{S}$ provides a Higgs boson $h$ with SM-like tree-level couplings to gauge bosons and fermions. However, there is still a possibility of deviation from the SM in loop couplings. The second doublet, $\Phi_{D}$, is inert and contains four dark (inert) scalars $H, A, H^{ \pm}$. The lightest particle from $\Phi_{D}$ is stable due to conserved $D$ symmetry and is a good DM candidate. We choose $M_{H}<M_{A, H^{ \pm}}{ }^{1}$

The properties of the IDM can be described by the masses of scalar particles and their couplings: $\lambda_{345}=\lambda_{3}+\lambda_{4}+\lambda_{5}$ is related to Higgs-DM triple and quartic couplings, $h H H$ and $h h H H$, while $\lambda_{3}$ gives $h H^{+} H^{-}$and $\lambda_{2}$ : a self-coupling $H H H H$. Parameters of the IDM have to fulfill various theoretical and experimental constraints. We use vacuum stability conditions which ensure the potential is bounded from below. We also demand, that the state (1.1) is the global, and not just a local minimum [2]. Parameters of the potential should also fulfill perturbative unitarity bounds [4]. The above conditions together with the value of the Higgs boson mass, $M_{h}=125 \mathrm{GeV}$, provide the following constraints for the parameters of the potential [5]:

$\lambda_{1}=0.258, m_{22}^{2} \lesssim 9 \cdot 10^{4} \mathrm{GeV}^{2}, \lambda_{3}, \lambda_{345}>-\sqrt{\lambda_{1} \lambda_{2}} \geqslant-1.47, \lambda_{2}^{\max }=8.38$.

Masses of dark particles are constrained by the LEP measurements and EWPT to be [6]: $M_{H^{ \pm}}+M_{H}>M_{W}, M_{H^{ \pm}}+M_{A}>M_{W}, M_{H}+M_{A}>M_{Z}, 2 M_{H^{ \pm}}>M_{Z}, M_{H^{ \pm}}>70 \mathrm{GeV}$ with an excluded region where simultaneously: $M_{H}<80 \mathrm{GeV}, M_{A}<100 \mathrm{GeV}$ and $\delta_{A}>8 \mathrm{GeV}$. We use mass differences $\delta_{A, \pm}=M_{A, H^{ \pm}}-M_{H}$.

There are three regions of $M_{H}$ that fulfill the above constraints and are in agreement with the WMAP measurements of relic density: (i) light DM particles with mass $\lesssim 10 \mathrm{GeV}, M_{A} \approx M_{H^{ \pm}}=$ $100 \mathrm{GeV}$, (ii) medium DM mass of $40-150 \mathrm{GeV}$ with $\delta_{A}<8 \mathrm{GeV}$ or $\delta_{A} \approx \delta_{H^{ \pm}} \approx(50-200) \mathrm{GeV}$ and (iii) heavy DM with mass $\gtrsim 500 \mathrm{GeV}$ and $\delta_{A} \approx \delta_{H^{ \pm}} \approx 1 \mathrm{GeV}$ [7].

\section{The diphoton decay rate $R_{\gamma \gamma}$ in the IDM}

$R_{\gamma \gamma}$ is the ratio of the diphoton decay rate of the Higgs particle $h$ observed at the LHC to the SM prediction. Deviation from $R_{\gamma \gamma}=1$ would be a sign of physics beyond the SM. The current

\footnotetext{
${ }^{1}$ Choosing $A$ as the lightest $D$-odd particle corresponds to $\lambda_{5} \rightarrow-\lambda_{5}$. Charged scalar as a DM candidate is excluded by positivity and perturbativity conditions $[3,2]$.
} 
measured values $R_{\gamma \gamma}=1.65 \pm 0.24(\mathrm{stat})_{-0.18}^{+0.25}$ (syst) for ATLAS and $R_{\gamma \gamma}=0.79_{-0.26}^{+0.28}$ for CMS [8] are in $2 \sigma$ agreement with $R_{\gamma \gamma}=1$. However, a deviation from the SM value is still possible.

The ratio $R_{\gamma \gamma}$ in the IDM is given by:

$$
R_{\gamma \gamma}:=\frac{\sigma(p p \rightarrow h \rightarrow \gamma \gamma)^{\mathrm{IDM}}}{\sigma(p p \rightarrow h \rightarrow \gamma \gamma)^{\mathrm{SM}}} \approx \frac{\Gamma(h \rightarrow \gamma \gamma)^{\mathrm{IDM}}}{\Gamma(h \rightarrow \gamma \gamma)^{\mathrm{SM}}} \frac{\Gamma(h)^{\mathrm{SM}}}{\Gamma(h)^{\mathrm{IDM}}},
$$

where $\Gamma(h)^{\mathrm{SM} / \mathrm{IDM}}$ are the total decay widths of $h$ in the SM and the IDM, while $\Gamma(h \rightarrow \gamma \gamma)^{\mathrm{SM} / \mathrm{IDM}}$ are the respective partial decay widths for $h \rightarrow \gamma \gamma$. In the IDM two sources of deviation from $R_{\gamma \gamma}=1$ are possible. First is a contribution from $H^{ \pm}$loop to the partial decay width $[9,10]$ :

$$
\Gamma(h \rightarrow \gamma \gamma)^{\mathrm{IDM}}=\frac{G_{F} \alpha^{2} M_{h}^{3}}{128 \sqrt{2} \pi^{3}}\left|\mathscr{M}^{\mathrm{SM}}+\delta \mathscr{M}^{\mathrm{IDM}}\left(M_{H^{ \pm}}, \lambda_{3}\right)\right|^{2}
$$

where $\mathscr{M}^{\mathrm{SM}}$ is the SM amplitude and $\delta \mathscr{M}^{\mathrm{IDM}}$ is the $H^{ \pm}$contribution [9]. The interference between $\mathscr{M}^{\mathrm{SM}}$ and $\delta \mathscr{M}^{\mathrm{IDM}}$ can be either constructive or destructive, leading to an enhancement or reduction of $R_{\gamma \gamma}$ with respect to the SM value. The second source of deviations are possible invisible decays $h \rightarrow H H, A A$, that change the total decay width $\Gamma^{\mathrm{IDM}}(h)$ with respect to the SM case. If $h$ can decay invisibly then those channels dominate over the SM decay channels and $R_{\gamma \gamma}$ is always below the SM value [11, 10]. For $M_{A}, M_{H}>M_{h} / 2$ the invisible channels are closed, and $R_{\gamma \gamma}>1$ is possible.

$R_{\gamma \gamma}$ depends only on the masses of the dark scalars and $\lambda_{345}$ (or $\lambda_{3}$ ), so setting a lower bound on $R_{\gamma \gamma}$ leads to upper and lower bounds on $\lambda_{345}$ as functions of $M_{H, A, H^{ \pm}}$. Below we summarize results obtained in $[12,13]$ :

$H H, A A$ decay channels open If $M_{H}, M_{A}<M_{h} / 2$ the invisible decay channels have stronger influence on the value of $R_{\gamma \gamma}$ than the contribution from the $H^{ \pm}$loop [11]. Demanding that $R_{\gamma \gamma}>$ 0.7 gives values of $\lambda_{345}$ typically in range $(-0.04,0.04)$. Larger $R_{\gamma \gamma}$ allows only for even smaller $\left|\lambda_{345}\right|$ and also sets limits on the mass difference $\delta_{A}[13]$.

$A A$ decay channel closed When the $A A$ decay channel is closed, $M_{A}>M_{h} / 2$, the $H^{ \pm}$contribution becomes more relevant. If $R_{\gamma \gamma}>0.7$ allowed values of $\left|\lambda_{345}\right|$ are of order 0.02. Larger value of $R_{\gamma \gamma}$ leads to smaller allowed values of $\lambda_{345}$. In the case of $R_{\gamma \gamma}>0.9$ it is not possible to obtain the requested value of $R_{\gamma \gamma}$ for any value of $\lambda_{345}$ if $M_{H} \lesssim 45 \mathrm{GeV}$ [13].

Invisible decay channels closed If $M_{A}, M_{H}>M_{h} / 2$, the invisible channels are closed and the only modification to $R_{\gamma \gamma}$ comes from the $H^{ \pm}$loop (2.2). Enhancement in $R_{\gamma \gamma}$ is possible when $\lambda_{3}<0[10,11]$. Even a small deviation from $R_{\gamma \gamma}=1$ requires a relatively large $\lambda_{345}$, if $\delta_{H^{ \pm}}$ is of order $(50-100) \mathrm{GeV}$. Small values of $\left|\lambda_{345}\right|$ are allowed only if $\delta_{H^{ \pm}}$is small [13]. Also, unitarity and positivity limits on $\lambda_{3}$ and $\lambda_{345}$ constrain the allowed values of $M_{H^{ \pm}}$and $M_{H}$ for a given value of $R_{\gamma \gamma}$. For $R_{\gamma \gamma}^{\max }=1.01$ masses of $M_{H^{ \pm}} \gtrsim 700 \mathrm{GeV}$ are excluded, $R_{\gamma \gamma}^{\max }=1.02$ forbids $M_{H^{ \pm}} \gtrsim 480 \mathrm{GeV}$, while $R_{\gamma \gamma}>1.2$ gives $70 \mathrm{GeV}<M_{H^{ \pm}}<154 \mathrm{GeV}$ [11].

\subsection{Combining $R_{\gamma \gamma}$ and relic density constraints on DM}

Combining the limits on the $\lambda_{345}$ parameter obtained from $R_{\gamma \gamma}$ with those coming from the requirement that $\Omega_{D M} h^{2}$ is in agreement with the WMAP measurements: 




(a) $h \rightarrow H H$ channel open

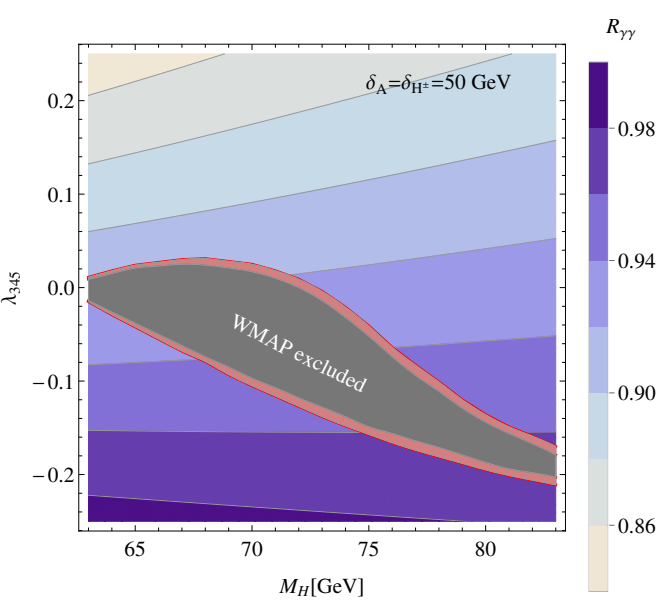

(b) $h \rightarrow H H$ channel closed

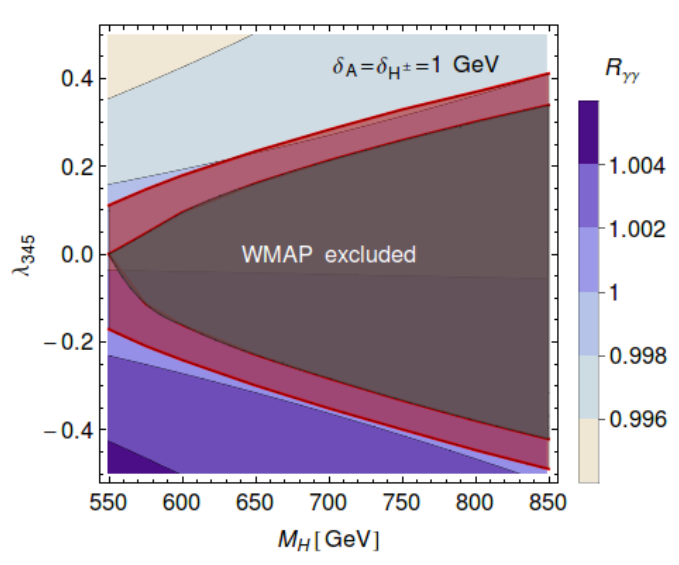

(c) high DM mass

Figure 1: Comparison of the values of $R_{\gamma \gamma}$ and region allowed by $\Omega_{D M} h^{2}$ measurements for the medium DM mass with (a) $H H$ invisible channel open and $M_{A}=M_{H^{ \pm}}=120 \mathrm{GeV}$, (b) with $H H$ invisible channel closed and $\delta_{A}=\delta_{H^{ \pm}}=50 \mathrm{GeV}$, (c) heavy DM particles with $\delta_{A}=\delta_{H^{ \pm}}=1 \mathrm{GeV}$. Red bound: region in agreement with WMAP. Grey area: excluded by WMAP.

$$
0.1018<\Omega_{D M} h^{2}<0.1234
$$

allows us to exclude parts of the IDM parameter space.

Low DM mass In the IDM the low DM mass region corresponds to $M_{H} \lesssim 10 \mathrm{GeV}$. To provide the proper relic density $\left|\lambda_{345}\right|$ has to be large, for example $\left|\lambda_{345}\right|=(0.35-0.41)$ for the CDMS-II favoured mass $M=8.6 \mathrm{GeV}$. The coupling allowed by $R_{\gamma \gamma} \sim 0.7$, i.e. $\left|\lambda_{345}\right| \sim 0.02$, is of an order of magnitude smaller than needed for $\Omega_{D M} h^{2}$. The low DM mass region cannot be accommodated in the IDM with recent LHC results.

Medium DM mass: invisible decay channels open We first consider the case with $M_{A}=M_{H^{ \pm}}=$ $120 \mathrm{GeV}$ and $M_{h} / 2>M_{H}>50 \mathrm{GeV}$, presented in figure 1a. For a large portion of the parameter 
space limits for $\lambda_{345}$ from $R_{\gamma \gamma}$, even for the least stringent case $R_{\gamma \gamma}>0.7$, cannot be reconciled with the WMAP-allowed region, where $\left|\lambda_{345}\right| \sim 0.1$, excluding $M_{H} \lesssim 53 \mathrm{GeV}$.

Medium DM mass: invisible decay channels closed Here we choose $\delta_{H^{ \pm}}=\delta_{A}=50 \mathrm{GeV}$ and $83 \mathrm{GeV}>M_{H}>M_{h} / 2$. Figure $1 \mathrm{~b}$ gives the WMAP-allowed range with the corresponding values of $R_{\gamma \gamma}\left|\lambda_{345}\right|$ that lead to the proper relic density are in general larger than in the case of $M_{H}<M_{h} / 2$. This region of $M_{H}$ is consistent with $R_{\gamma \gamma}<1$. If the IDM is the source of all DM in the Universe and $M_{H} \approx(63-83) \mathrm{GeV}$ then the maximal value of $R_{\gamma \gamma}$ is around 0.98. A subdominant DM candidate, which corresponds to larger $\lambda_{345}$, is consistent with $R_{\gamma \gamma}>1$.

High DM mass $\quad$ It is possible to obtain $R_{\gamma \gamma}>1$ and be with agreement with WMAP for $M_{H} \gtrsim 500$ $\mathrm{GeV}$ and $\delta_{A}=\delta_{H^{ \pm}}=1 \mathrm{GeV}$, as shown in figure 1c, although deviation from $R_{\gamma \gamma}=1$ is very small.

\subsection{Comparison with direct and indirect detection limits}

Comparison with XENON100 results In the IDM the DM-nucleon scattering cross-section $\sigma_{D M, N}$ is given by:

$$
\sigma_{\mathrm{DM}, \mathrm{N}}=\frac{\lambda_{345}^{2}}{4 \pi M_{h}^{4}} \frac{m_{N}^{4}}{\left(m_{N}+M_{H}\right)^{2}} f_{N}^{2},
$$

with $M_{h}=125 \mathrm{GeV}, m_{N}=0.939 \mathrm{GeV}$ and $f_{N}=0.326$ being the universal Higgs-nucleon coupling. ${ }^{2}$

Invisible channel open Value of the $\lambda_{345}$ coupling is essential for the value of $\sigma_{D M, N}$ in the IDM and so we translate the limits for $\lambda_{345}$ obtained from $R_{\gamma \gamma}$ measurements to $\left(M_{H}, \sigma_{D M, N}\right)$ plane, used in direct detection experiments. Exclusion bounds for cases $R_{\gamma \gamma}>0.7,0.8$ are shown in figure 2, along with the XENON10/100 limits [15]. If $H$ constitutes $100 \%$ of DM in the Universe, then the limits set by $R_{\gamma \gamma}$ are much stronger than the ones provided by XENON10/100 experiments for $M_{H} \lesssim 20 \mathrm{GeV}$. Even for $R_{\gamma \gamma}>0.7$ it provides stronger or comparable limits for $\sigma_{D M, N}$ for $M_{H} \lesssim 60$ $\mathrm{GeV}$.

Invisible channels closed If $H$ constitute $100 \%$ of DM in the Universe, then the $\sigma_{D M, N}$ measurements performed by the direct detection experiments limit the $\lambda_{345}$ parameter, which is also constrained by the $R_{\gamma \gamma}$ value (figure 3). For given $M_{H}, M_{H^{ \pm}}$one can test the compability between the two limits, and figure 3 shows that $R_{\gamma \gamma}>1$ and agreement with XENON100 require almost degenerated masses of $H$ and $H^{ \pm}$. If $\delta_{H^{ \pm}}$is larger then $R_{\gamma \gamma}$ requires larger $\lambda_{345}$, and that violates the XENON100 bounds.

Comparison with the indirect detection limits The current best limits on DM annihilation in the IDM, come from the Fermi-LAT measurements. They exclude the generic WIMP candidates that annihilate mainly into $b \bar{b}$ and reproduce the observed $\Omega_{D M} h^{2}$ for $M_{D M} \lesssim 25 \mathrm{GeV}$ [16]. ${ }^{3}$ The combined $\Omega_{D M} h^{2}$ and $R_{\gamma \gamma}$ analysis excludes masses of DM in the IDM below $53 \mathrm{GeV}$ if $R_{\gamma \gamma}>0.7$ and thus gives stronger limits on the allowed values of masses in the IDM than the ones currently obtained from the indirect detection experiments [13].

\footnotetext{
${ }^{2}$ There is no agreement on the value of $f_{N}$, here we consider the middle value of $0.14<f_{N}<0.66$ [14].

${ }^{3}$ Independent analyses give slightly stronger limits, excluding generic WIMPs with $M_{D M}$ less than $40 \mathrm{GeV}$ [17].
} 


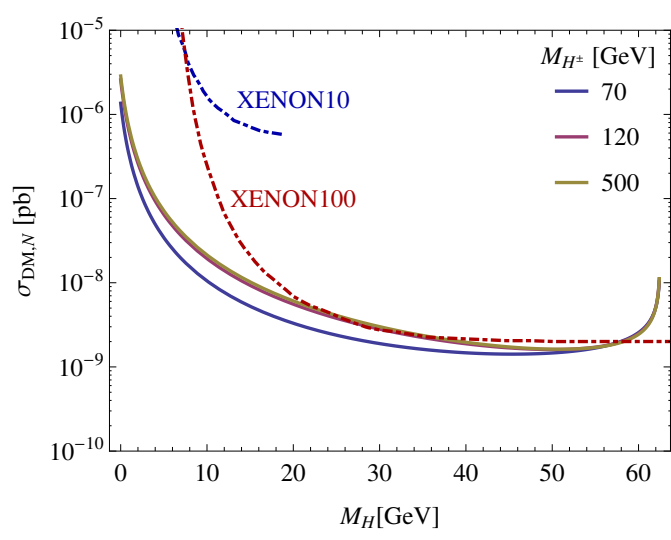

(a) $R_{\gamma \gamma}>0.7$

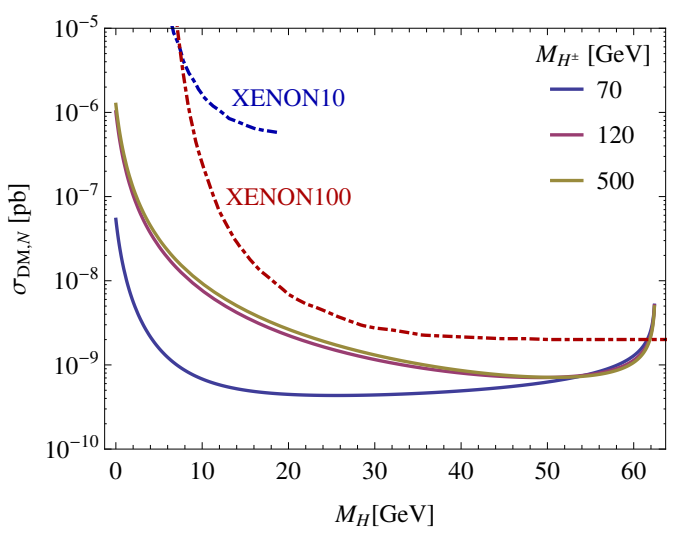

(b) $R_{\gamma \gamma}>0.8$

Figure 2: Upper limit on $\sigma_{\mathrm{DM}, N}$ with $f_{N}=0.326$ coming from the requirement that (a) $R_{\gamma \gamma}>0.7$, (b) $R_{\gamma \gamma}>0.8$, for the case when the $h \rightarrow A A$ channel is closed. For comparison also the upper bounds set by XENON10 and XENON100 are shown.

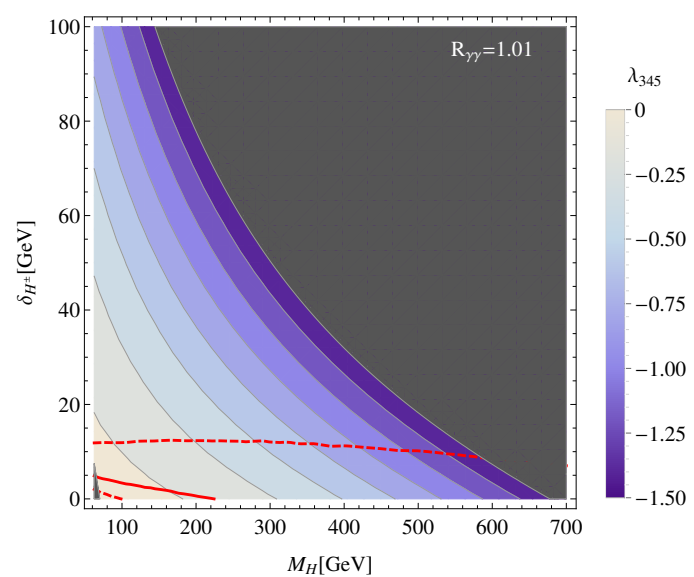

(a) $R_{\gamma \gamma}=1.01$

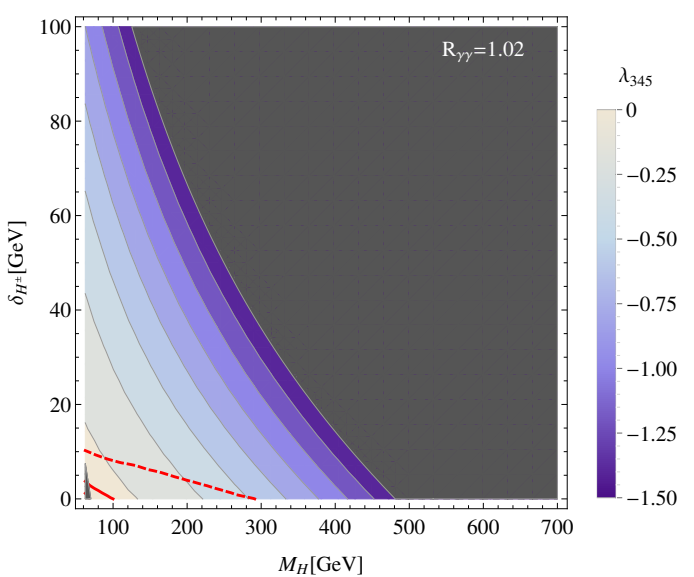

(b) $R_{\gamma \gamma}=1.02$

Figure 3: Allowed regions in $\left(M_{H}, \delta_{H^{ \pm}}\right)$plane for: (a) $R_{\gamma \gamma}=1.01$, (b) $R_{\gamma \gamma}=1.02$. Dark grey region is excluded due to LEP bounds (left lower corner) and the vacuum stability/unitarity constraints (right upper corner). Red lines show bounds from XENON100 (solid for $f_{N}=0.326$, dashed for $f_{N}=0.14$ and $\left.f_{N}=0.66\right)$ - region above this line is excluded, if we assume that the dark scalar $H$ constitutes all dark matter relic density.

\section{Summary}

The DM candidate from the IDM is consistent with the WMAP results on the DM relic density. Measurements of the diphoton ratio $R_{\gamma \gamma}$ at the LHC set strong limits on masses of the DM and other dark scalars, and their self-couplings.

We can exclude the low DM mass region in the IDM, i.e. $M_{H} \lesssim 10 \mathrm{GeV}$, as values of $\left|\lambda_{345}\right|$ needed for the proper $\Omega_{D M} h^{2}$ are an order of magnitude larger than those allowed by $R_{\gamma \gamma}>0.7$. 
In the medium mass region it is possible to have $R_{\gamma \gamma}<1$ and all DM in the Universe explained by the IDM. If $R_{\gamma \gamma}>1$ then $H$ may be a subdominant DM candidate. For heavy DM particles it is possible to obtain $R_{\gamma \gamma}>1$ and fulfill WMAP bounds, although deviation from $R_{\gamma \gamma}=1$ is small.

We conclude that $R_{\gamma \gamma}$ sets limits on the DM-nucleon scattering cross-section in the low and medium DM mass region, which are stronger or comparable with the results obtained both by the XENON100 and Fermi-LAT experiments.

\section{References}

[1] Q.-H. Cao et al, Phys.Rev., vol. D76, p. 095011, 2007; R. Barbieri et al, Phys. Rev., vol. D74, p. $015007,2006$.

[2] I. Ginzburg et al, Phys.Rev., vol. D82, p. 123533, 2010; D. Sokolowska, Acta Phys.Polon., vol. B42, p. 2237, 2011.

[3] M. Krawczyk and D. Sokolowska, arXiv:0911.2457 [hep-ph].

[4] S. Kanemura et al, Phys.Lett., vol. B313, pp. 155-160, 1993.

[5] B. Swiezewska, 2012, arXiv:1209.5725 [hep-ph].

[6] E. Lundstrom et al, Phys.Rev., vol. D79, p. 035013, 2009.

[7] E. M. Dolle and S. Su, Phys.Rev., vol. D80, p. 055012, 2009; E. Dolle, X. Miao, S. Su and B. Thomas, Phys. Rev. D 81, 035003 (2010); C. Arina, F. S. Ling and M. H. G. Tytgat, JCAP 0910 (2009) 018; T. Hambye and M. H. G. Tytgat, Phys. Lett. B 659 (2008) 651; E. Nezri, M. H. G. Tytgat and G. Vertongen, JCAP 0904 (2009) 014; S. Andreas, M. H. G. Tytgat and Q. Swillens, JCAP 0904 (2009) 004; S. Andreas, T. Hambye and M. H. G. Tytgat, JCAP 0810 (2008) 034; L. Lopez Honorez, E. Nezri, J. F. Oliver and M. H. G. Tytgat, JCAP 0702 (2007) 028.

[8] ATLAS Collaboration, ATLAS-CONF-2013-012; C. Mariotti, Talk given at CERN, 15.04.2013.

[9] A. Djouadi, Phys.Rept., vol. 459, pp. 1-241, 2008; Phys.Rept., vol. 457, pp. 1-216, 2008.

[10] A. Arhrib et al, Phys.Rev., vol. D85, p. 095021, 2012.

[11] B. Swiezewska and M. Krawczyk, Phys. Rev. D 88 (2013) 035019.

[12] M. Krawczyk, D. Sokolowska and B.Swiezewska, J. Phys. Conf. Ser. 447 (2013) 012050.

[13] M. Krawczyk, D. Sokolowska, P. Swaczyna and B. Swiezewska, JHEP 1309 (2013) 055.

[14] S. Andreas, M. H. Tytgat, and Q. Swillens, JCAP, vol. 0904, p. 004, 2009.

[15] E. Aprile et al., Phys.Rev.Lett., vol. 109, p. 181301, 2012.

[16] M. Ackermann et al., Phys. Rev. Lett. 107 (2011) 241302; M. Ackermann et al., Phys. Rev. D 86, 022002 (2012).

[17] K. N. Abazajian, P. Agrawal, Z. Chacko and C. Kilic, Phys. Rev. D 85 (2012) 123543; A. Geringer-Sameth and S. M. Koushiappas, Phys. Rev. Lett. 107 (2011) 241303. 